\title{
New Parametric Approach for the General Lorenz System
}

\author{
Lazhar Bougoffa ${ }^{1}$ and Smail Bougouffa ${ }^{2}$ \\ ${ }^{1}$ Al Imam Mohammad Ibn Saud Islamic University (IMSIU), Faculty of Science, \\ Department of Mathematics, P.O. Box 90950, Riyadh 11623, Saudi Arabia \\ ${ }^{2}$ Department of Physics, Faculty of Science, Taibah University, P.O. Box 30002, \\ Madina, Saudi Arabia \\ E-mail: ${ }^{1}$ bougoffa@hotmail.com, ${ }^{2}$ sbougouffa@hotmail.com and \\ sbougouffa@taibahu.edu.sa
}

\begin{abstract}
We are concerned with the study of the system of coupled equations of motion for a system of two-level atoms interacting with a single-mode field in the laser cavity on resonance. The passage from the equation of motion to the Lorenz equations is established. Some theoretical aspects of the slowly time -varying phenomena in cavities are discussed and the linear stability analysis is presented. A new approach to solve this nonlinear coupled differential equations is explored and its generalization to the case of variable coefficients is performed.

PACS numbers: $\quad$ 55.45.Pq, 05.40.Ca,32.80.Pj, 42.50.Vk, 42.50.Hz, 42.50.Lc
\end{abstract}

Keywords: Optical Bloch equations; Field-atom interactions; coupled differential equations; Navier-Stockes equations; Lorenz equations; Chaos; Steady-state solutions; Time-varying phenomena; Hopf bifurcation; Single-mode laser. 


\section{Introduction}

In many problems in quantum optics, dynamical chaos plays an important role. These include, for example, nonlinear systems and deterministic chaos in lasers. In the last case, it has been shown that, even when the laser is treated semiclassically by deterministic equations, the equations are capable of exhibiting quasi-periodic and even chaotic behavior, but this has nothing to do with quantum fluctuations [1 3]. In general, the study of the time-varying phenomena in cavities shows how various cavity instabilities can be lead to dynamical chaos, which occurs in many coupled nonlinear problems [4-7]. One of the simplest but popular models in dynamical chaos is the Lorenz model, which illustrates one of the powerful aspects of the study of chaos: generic features that are learned from one field - here hydrodynamics- can readily be adapted to another - laser physics. Haken [8] showed the existence of an isomorphism between the Bloch equations and the Lorenz equations. Of course, the Lorenz system is of considerable interest in its own right. This has been true from the beginning when it used in the chaos problems [9] nearly forty years ago, to a recent emergence of a computer assisted proof that such comportment is present for this system [10]. For more on the Lorenz system, see [11,12] and references therein. We are interested, however, in the evolution of the variable associated with the nonlinear systems. This requires us to obtain the equations of motion for the nonlinear systems of interest and solve them. In order to treat these nonlinear equations and their stability of their solutions, we briefly present the correspondence between the Bloch equations for a system of identical two-level atoms interacting with a single-mode field in the laser cavity on resonance and the Lorenz equations in hydrodynamic. Then we discuss some of the characteristics of solutions of these nonlinear coupled differential equations equations which can not always be exactly separated. It may, however, be worthwhile if the physical models can be constructed in such manner that the coupled system can either be solved analytically or transformed into another system in which the equations are decoupled and solved separately. In this work we attempt to present a new analytic approach to solve this system and then discus the possibility to extend this approach for the case of variable coefficients. Within this scope this paper begins in section 2 with setting up the Bloch equations for a system of two-level atoms interacting with a single-mode field in the laser cavity on resonance. In section 3 we establish the relationship to the Lorenz equations. Section 4 presents some aspects and features of the Lorenz equations. Section 5 shows the linearization approach where the system can be treated using the separation approach discussed in 13 . In section 6 , we explore a new approach to solve analytically the Lorenz system for a particular class of initial conditions. In Section 7, we extend our approach to include the solutions of the Lorenz system with variable coefficients, and we conclude in last section with some remarks and possible extension of the domain of investigation. 


\section{The single Mode Laser Equations}

In the previous papers [13, 14] we have explored various aspects of the optical Bloch equations (OBE) which are widely used for describing dynamics in a system consisting of molecules, electromagnetic waves, thermal bath, and the near-resonant interaction of spin or atomic transition with radiation field. In general, the optical Bloch equations are treated in steady-state, to provide the mean radiative forces for a two- and three-level atom. Contrary to the common steady state approximation, the dynamics and transient optical effects on atomic motion can be considered by solving the complete system in some circumstances. In the framework of the semiclassical approximation and with the rotating wave approximation [4,5], for the pure radiative decay which is defined by an upper-to-lower-level transition with the dipole constant $1 / T_{1}$, the motion equations of the components of the Bloch vector $(U, V, W)$ form a system of coupled differential equations. For central tuning, it can be assumed that $U=0$ and the two remaining equations are coupled to slowly-varying amplitude equation. Then the system can be read as

$$
\begin{aligned}
& \dot{V}=-\frac{1}{T_{2}} V-\Omega(\mathbf{r}) W, \\
& \dot{W}=\Omega(\mathbf{r}) V-\frac{1}{T_{1}}(W+1), \\
& \dot{\Omega}=-\frac{1}{T_{c}} \Omega+\omega g V,
\end{aligned}
$$

where for simplicity, we assume that the field frequency $\omega$ is resonant with the atomic transition $\omega_{0}$, i.e., the detuning $\delta=0$ and we lump all rapid spatial dependence of the field into the Rabi frequency $\Omega(\mathbf{r})$ that is now a function of position in general. The "dot" denotes the time derivative $d / d t, \frac{1}{T_{c}}$ is the cavity damping rate and $g$ is the coupling rate between the cavity field and the atom. In keeping with the literature on NMR we use $T_{2}$ for the induced dipole decay and $T_{1}$ for the probability difference decay time.

The usual laser threshold, in which the gain rate equals the loss rate, matches to the condition

$$
\omega g=\frac{1}{T_{c} T_{2}} .
$$

These coupled equations are known in literature [6] as the Lorenz-Maxwell equations and form a nonlinear system which can not always be exactly separated. Their nonlinear character can lead to chaotic instabilities (classical chaos) in atomic and field dynamics. On the other hand, this system is isomorphic to the Lorenz equations as it was demonstrated by Haken [7, 8, so that a laser governed by these equations is capable of exhibiting the same chaotic behavior. In the following section we shall present the passage form these laser equations to the Lorenz equations, which demonstrate that much more complexity is possible once we have three coupled variables. 


\section{Passage to the Lorenz equations}

The original purpose of the Lorenz equations was to introduce a simple model of the convection instability, which occurs when a fluid is heated from below and kept at a constant temperature from above [4]. For small temperature gradients, the fluid remains quiescent, but it starts a macroscopic motion as the gradient approaches a critical value. The heated parts of the fluid expand, move up by buoyancy, cool and fall back to the bottom: a regular spatial pattern appears out of a completely homogeneous state.

The motion of the fluid was described by the nonlinear, partial differential NavierStockes equations of hydrodynamics, which are first undertaken in [15, 16]. In later, by introducing a special Fourier decomposition of the velocity and temperature fields in the fluid, Lorenz derived a set of truncated dimensionless equations coupling just one component $x$ of the velocity field to two components $y$ and $z$ of the temperature field [7,17]. The Lorenz equations have the following form:

$$
\begin{aligned}
& \dot{x}=-a x+a y, \\
& \dot{y}=-y-x z+r x, \\
& \dot{z}=-b z+x y,
\end{aligned}
$$

where $b>0, a>0$, and $r>1$. The constants $a, r, b$ are related to the physical parameters. For example, in fluid dynamics $a$ is the Prandtl number, $r=R / R_{c}$, where $R$ is the Rayleigh number and $R_{c}$ the critical Rayleigh number [4]. $b=4 \pi^{2} /\left(\pi^{2}+k_{1}^{2}\right)$, where $k_{1}$ is a dimensionless wave number. These equations are ordinary differential equations and hold within two nonlinearities in the form $x z$ and $x y$. To the great surprise of many mathematicians and physicists these equations can have solutions which are quite irregular. These solutions were found numerically.

In order, to establish the correspondence between the Lorenz equations (3) and the single-mode laser equations (11), we find the steady-state solutions of Eqs. (11) which can be obtained by putting the time derivatives equal to zero. We then determine the following solutions in which the field is non-zero,

$$
\begin{aligned}
W_{s s} & =-\frac{1}{\omega g T_{c} T_{2}}, \\
V_{s s} & =\frac{1}{\omega g T_{c}} \sqrt{\frac{1}{T_{1} T_{2}}\left(\omega g T_{c} T_{2}-1\right)}, \\
\Omega_{s s} & =\sqrt{\frac{1}{T_{1} T_{2}}\left(\omega g T_{c} T_{2}-1\right)},
\end{aligned}
$$

provide that $\omega g T_{c} T_{2} \geq 1$. It is convenient now to introduce normalized variables in terms of these steady-state expressions, and make the following substitution

$$
x=\kappa \Omega / \Omega_{s s}, \quad y=\kappa V / V_{s s}, \quad z=-(W+1) / W_{s s},
$$

where

$$
\kappa=\sqrt{\frac{T_{2}}{T_{1}}\left(\omega g T_{c} T_{2}-1\right)} .
$$


We attain exactly the equations (3), except that now

$$
a=\frac{T_{2}}{T_{c}}, \quad b=\frac{T_{2}}{T_{1}}, \quad r=\omega g T_{c} T_{2}
$$

and time is now in units of $T_{2}$. We note here that these equations can give a detailed account of turbulence motion and chaos when $a>b+1$. This condition correspond to the following condition on the damping constants

$$
\frac{1}{T_{c}}>\frac{1}{T_{2}}+\frac{1}{T_{1}}
$$

which indicates that the atomic decay rates can not exceed the cavity rate, which is in contradiction with the usual condition that was assumed in semiclassical theory of laser in order to make an adiabatic elimination of the atomic variables. It is now clear that chaos can not show up in semiclassical treatment of laser problem; it requires a bad cavity, in which the spectral width of the cavity field exceeds the atomic homogeneous linewidth [5]. The parameter $\omega g T_{2}$ represents the gain rate of the laser, which equals the loss rate $1 / T_{c}$ at the laser threshold. Then the quantity $\omega g T_{2} T_{c}$ measures how many times the gain exceeds the loss. In the following we shall pass to present some features of Lorenz equations.

\section{Some features of Lorenz equations}

We are not concerned directly here with the subject of fluid flow, but we shall present some important features of the Lorenz equations which are isomorphic to the single mode laser equations. In addition to they are nonlinear coupled equations, sensitive to the initial conditions and all their trajectories are eventually confined to paths of zero volume, these equations have some important theoretical aspects, which can briefly summarized in the following points:

A steady state of a system is a point in phase space from which the system will not change in time, once that state has reached. In other words, it is a point, $(x, y, z)$, such that the solution does not change, or where

$$
\dot{x}=0, \quad \dot{y}=0 \quad \text { and } \quad \dot{z}=0 .
$$

This point is usually referred to as a stationary point of the system and is called a fixed point. By setting the time derivatives equal to zero in the Lorenz equations (13, 14, 15), and solving the resulting system we find three possible steady states, namely the stationary points

$$
C^{0}=(0,0,0), \quad C^{ \pm}=( \pm \sqrt{b(r-1)}, \pm \sqrt{b(r-1)}, r-1),
$$

so that there is only one fixed point when $0 \leq r \leq 1$ but all three fixed points are present when $r>1$ which corresponds to the laser threshold [4]. As $r \rightarrow 1, C^{ \pm}$coalesce with the origin in a pitchfork bifurcation [12].

The stability of the non-linear system is almost always similar to that the linearized systems near the fixed points. Then, the stability of the fixed points of the Lorenz 
equations can be established by linear stability analysis for more details see [12, 18]. The origin $(0,0,0)$ is a stable fixed point for $r<1$; that is it attracts nearby solutions to itself and it becomes unstable for $r>1$. In this last case the two other fixed points in turn become unstable when $a>b+1$ and $r>a(a+b+3) /(a-b-1)$. This is called a Hopf bifurcation [18, 19.

The right-hand side of the second inequality can be considered as a function of $a$ and it has a minimum when

$$
a=1+b+\sqrt{2(b+1)(b+2)}
$$

The minimum value of the function can be given by

$$
\min .=2 \sqrt{2(b+1)(b+2)}+3(b+1)+2,
$$

which depends only on the parameter $b$.

In the laser case, this minimum value represents the minimum value of the gain-to-lossratio, which shows that how large this has to be far for instability to be encountered, and reads

$$
\min .=2\left[2\left(\frac{T_{2}}{T_{1}}+1\right)\left(\frac{T_{2}}{T_{1}}+2\right)\right]^{1 / 2}+3\left(\frac{T_{2}}{T_{1}}+1\right)+2,
$$

The minimum gain-to-loss ratio is refereed to as the second laser threshold, depends on $T_{2} / T_{1}$, but always exceeds 9 .

The difficulty part of doing any theoretical analysis of the Lorenz equations is that they are non-linear. So, the system of non-linear equations can be approximated by a linear one, using a Taylor series around a fixed point. The basic idea is to replace the right hand side functions in the equations (3) with linear approximation about the fixed point, and then solve the resulting system of linear ordinary differential equations . The obtained linear system can be treated using our technique that discussed in references [13, 14].

\section{Linear stability treatment}

In order to examine whether the steady-state solution for the fixed point given by (9) represents a stable state, we linearize the Lorenz equations in the neighborhood of the fixed point by using Taylor series of functions of multi-variables: $f(x, y, z)$. If we linearize a function $f(x, y, z)$ about $\left(x_{s}, y_{s}, z_{s}\right)$ we obtain the approximation

$$
\begin{aligned}
f(x, y, z) \approx & f\left(x_{s}, y_{s}, z_{s}\right)+f_{x}\left(x_{s}, y_{s}, z_{s}\right)(x-x s)+ \\
& +f_{y}\left(x_{s}, y_{s}, z_{s}\right)\left(y-y_{s}\right)+f_{z}\left(x_{s}, y_{s}, z_{s}\right)\left(z-z_{s}\right) .
\end{aligned}
$$

Applying this relation to the right hand side function for each of the ordinary differential

equations in (3), then the linearization of the original equations about the origin $C^{0}$ yields

$$
\begin{aligned}
& \dot{x}=-a x+a y, \\
& \dot{y}=-y+r x, \\
& \dot{z}=-b z,
\end{aligned}
$$


Hence, the z-motion decouples, leaving the two first equations linearly coupled. The solution to these equations is given by

$$
h(t)=C_{h 1} e^{\lambda_{1} t}+C_{h 2} e^{\lambda_{2} t}+C_{h 3} e^{\lambda_{3} t}, \quad h=x, y, z .
$$

The $C_{h i}$ 's are constants that are determined by the initial conditions of the problem, and $\lambda_{i}$ are the eigenvalues of the matrix of the coefficients of $\mathrm{x}, \mathrm{y}$, and $\mathrm{z}$ in the right hand side of equations (15), which are given by

$$
\lambda_{1}=-b, \quad \text { and } \quad \lambda_{2}, \lambda_{3}=\frac{1}{2}\left(-a-1 \pm \sqrt{(a-1)^{2}+4 a r}\right) .
$$

The stability of the non-linear system is almost always similar to that the linearized systems near the fixed points [20]. Then, the stability of the fixed points of the Lorenz equations can be established by linear stability analysis for more details see [12,18]. For $r<1$ all directions are incoming. The origin $(0,0,0)$ is a sink and stable node. If $r>1$, it attracts nearby solutions to itself and it becomes a 2D saddle point and unstable: two incoming and one outcoming directions. In this last case the two other fixed points in turn become unstable when $a>b+1$ and $r>r_{t}=a(a+b+3) /(a-b-1)$. This is called a Hopf bifurcation [18]. The bifurcations occur at:

* $r=1$, when the origin changes from stable to unstable, and two other fixed points appear.

* $r=r_{t}$, where the remaining two fixed points change from being stable to unstable.

When $r>1$, the same linearization process can be applied at the remaining two stationary points $C^{ \pm}$, which give an other system of equations:

$$
\begin{aligned}
& \dot{x}=-a x+a y, \\
& \dot{y}=-y+x \pm \sqrt{b(r-1)} \bar{z} \\
& \dot{\bar{z}}= \pm \sqrt{b(r-1)}(x+y)-b \bar{z}-2 b(r-1),
\end{aligned}
$$

where $\bar{z}=z-r+1$. These equations are similar to those of Bloch studied in [13]. The same treatment can be use here to obtain the solutions of this linear system. The eigenvalues of this system satisfy another characteristic equation:

$$
\lambda^{3}+(a+b+1) \lambda^{2}+(r+a) b \lambda+2 a b(r-1)=0 .
$$

In fact, the eigenvalues give us all the information we need to know about how the linearized solution behaves in time. It is possible that two of the eigenvalues can be complex numbers that if $\lambda_{2}=\lambda_{3}=\alpha \pm i \beta$ then the homogeneous solutions can be rearranged so that they are of the form

$$
h(t)=C_{h 1} e^{\lambda_{1} t}+C_{h 2} e^{\alpha t} \cos (\beta t)+C_{h 3} e^{\alpha t} \sin (\beta t), \quad h=x, y, \bar{z} .
$$

In terms of the asymptotic stability of the problem, we need to look at the asymptotic behavior of the solutions (20), as $t \rightarrow \infty$, from which several conclusions can be drawn:

(i) If the eigenvalues are real and at least one is positive, then the solutions will blow up as $t \rightarrow \infty$. In this case the linearized solutions are unstable. 
(ii) If the eigenvalues are real and negative, then the solutions will go to zero as $t \rightarrow \infty$. In this case the linearized solutions are stable.

(iii) If there is a complex conjugate pair of eigenvalues, $\alpha \pm i \beta$, then the solutions exhibit oscillatory behavior (with the emergence of the terms $\sin (\beta t)$ and $\cos (\beta t)$ ). If the real part, $\alpha$, of all eigenvalues is negative, the oscillations will decay in terms of time and the solutions are stable; if $\alpha>0$, then the oscillations will grow, and the solutions are unstable.

(iv) If the complex eigenvalues are pure imaginary, then the oscillations will neither increase nor decay in terms of time. The linearized solutions are periodic, and marginally stable.

It is a clear that the linear results apply only near the stationary points, and do not apply to all of the phase space. One difficulty that has not mentioned is that for values of $r>r_{t}$, the systems have oscillatory solutions, which are unstable. Linear theory does not reveal what happens when these oscillations become large. In order to study more closely the long-time behavior of the solution, we must recourse to another approach, which will be presented in the following parts to avoid the numerical treatments [21]. From the point of view experimental, Klische and Weiss [22] achieved in observing a sequence of pulsations of the light output, including successive period doubling and eventually chaos. Furthermore, Harrison et al. [23] succeeded in observing of instabilities leading to chaos in the emission from single mode homogeneous broadened single mode and multi-mode midinfrared Raman laser.

\section{Construction of exact parametric solutions of the Lorenz system}

In this section, we will present a direct approach to solve the Lorenz model with constant coefficients.

Multiplying both sides of the first equation of system (3) by $x$ we can get

$$
x y=\frac{1}{a}\left[x \frac{d x}{d t}+a x^{2}\right] .
$$

Substituting Eq. (21) into the third equation of system (3), we can obtain

$$
\frac{d}{d t}\left(z-\frac{1}{2 a} x^{2}\right)=-b\left(z-\frac{1}{b} x^{2}\right) \text {. }
$$

Assuming that $b=2 a$, thus Eq. (22) gives

$$
z-\frac{1}{b} x^{2}=c e^{-b t}
$$

where $c$ is a free parameter (constant of integration) that can be chosen equal zero for some class of initial conditions $z(0)-\frac{1}{b} x^{2}(0)=0$. 
Now, substituting Eq. (22) into the second differential equation of system (3), we obtain

$$
\frac{d y}{d t}=r x-y-\frac{1}{b} x^{3}
$$

Differentiating both sides of the first equation of system (3) with respect to $t$, we get

$$
\frac{d^{2} x}{d t^{2}}=-a \frac{d x}{d t}+a \frac{d y}{d t}
$$

Substituting Eq. (24) into Eq. (25), taking into account that $b=2 a$, and in view of

$$
a y=a x+\frac{d x}{d t}
$$

we have

$$
\frac{d^{2} x}{d t^{2}}+(a+1) \frac{d x}{d t}+a(1-r) x=-\frac{1}{2} x^{3} .
$$

Let $v=\frac{d x}{d t}$. Since

$$
\frac{d^{2} x}{d t^{2}}=\frac{d v}{d t}=\frac{d v}{d x} \frac{d x}{d t}=v \frac{d v}{d x}
$$

Thus

$$
v \frac{d v}{d x}=-(a+1) v+a(r-1) x-\frac{1}{2} x^{3}
$$

This is the Abel differential equation of the second kind.

The substitution $\xi=-(a+1) x$ guides the Abel equation to the simplified form

$$
v \frac{d v}{d \xi}-v=\phi(\xi)
$$

where the function $\phi(\xi)$ is defined parametrically by the relations

$$
\phi(\xi)=\frac{a(r-1)}{(a+1)^{2}} \xi-\frac{1}{2(a+1)^{4}} \xi^{3} .
$$

Once $v$ is found then we can get $x$ through $\frac{d x}{d t}=v(x)$.

Much more about the Abel equations and a large number of their solutions for various $\phi(\xi)$, can be found in standard textbooks, e.g. ( Polyamin and Zaitsev [24]). Particularly, the solution of the following equation

$$
v \frac{d v}{d \xi}-v=-\frac{2(m+1)}{(m+3)^{2}} \xi+A \xi^{m},
$$

is given in the parametric form as follows:

$$
\begin{aligned}
& \xi=\frac{m+3}{m-1} \alpha \tau E_{m}^{\frac{2}{m-1}}, \\
& v=\alpha E_{m}^{\frac{2}{m-1}}\left(R_{m} E_{m}+\frac{2}{m-1} \tau\right),
\end{aligned}
$$

where

$$
A= \pm \frac{m+1}{2}\left(\frac{m-1}{m+3}\right)^{m+1} \alpha^{1-m}
$$


New Parametric Approach for the General Lorenz System

$$
E_{m}=\int\left(1 \pm \tau^{m+1}\right)^{\frac{-1}{2}} d \tau-C_{1}
$$

and

$$
R_{m}=\sqrt{1 \pm \tau^{m+1}}
$$

Here $\tau$ is a parameter and $C_{1}$ is an arbitrary constant.

Combining Eqs. (30, 31) with Eqs. (32,37), we obtain

$$
\frac{a(r-1)}{(a+1)^{2}}=-\frac{2(m+1)}{(m+3)^{2}}
$$

and

$$
\frac{1}{2(a+1)^{4}}=\frac{m+1}{2}\left(\frac{m-1}{m+3}\right)^{m+1} \alpha^{1-m},
$$

where $m=3$. After some algebra, we obtain

$$
r=1-\frac{\alpha}{a} \text { and } \alpha= \pm 2\left(\frac{a+1}{3}\right)^{2} .
$$

Therefore our result can be reformulated as

Lemma 1 If $b=2 a$ and $r=1-\frac{\alpha}{a}$, where $\alpha= \pm 2\left(\frac{a+1}{3}\right)^{2}$. Then the solution $(x(t), y(t), z(t))$ of system (3) for a class of initial conditions $z(0)-\frac{1}{b} x^{2}(0)=0$ is given as follows:

$$
\frac{d x}{d t}=v(x)
$$

where $v$ is given in parametric form by

$$
\begin{aligned}
& \xi=3 \alpha \tau E_{3}, \quad \xi=-(a+1) x, \\
& v(\xi)=\alpha E_{3}\left(R_{3} E_{3}+\tau\right),
\end{aligned}
$$

where

$$
E_{3}=\int\left(1 \pm \tau^{4}\right)^{\frac{-1}{2}} d \tau-C_{1}
$$

and

$$
R_{3}=\sqrt{1 \pm \tau^{4}}
$$

Once $v$ is found then we can get $x(t)$ through $\frac{d x}{d t}=v(x)$,

$$
z(t)=\frac{1}{b} x^{2}(t)
$$

and $y(t)$ can be obtained from

$$
\frac{d y}{d t}=r x-y-x z
$$

that is

$$
y(t)=e^{-t} \int e^{t} \chi(t) d t+C_{2} e^{-t}, \chi(t)=r x(t)-x(t) z(t) .
$$




\section{The general Lorenz system with variable coefficients}

One of the main problem of mathematical physics appears when $a(t), b(t)$ and $r(t)$ are analytic functions and are added to the original system. The new problem, incorporating the above functions, is

$$
\left\{\begin{aligned}
\frac{d x}{d t} & =-a(t) x+a(t) y \\
\frac{d y}{d t} & =r(t) x-y-x z \\
\frac{d z}{d t} & =-b(t) z+x y .
\end{aligned}\right.
$$

A question which arises naturally is under what conditions on the functions $a(t), b(t)$ and $r(t)$ does the given system have an explicit solution?

In this section, we will propose a similar approach to solve the general Lorenz model. Proceeding as before, after some algebra we can get

$$
\frac{d}{d t}\left(z-\frac{1}{2 a(t)} x^{2}\right)=-b(t)\left(z-\frac{1}{b(t)}\left[1-\left(\frac{1}{2 a(t)}\right)^{\prime}\right] x^{2}\right) .
$$

where the prime means a first derivative respect to $t$. Assuming that

$$
\frac{1}{b(t)}\left[1-\left(\frac{1}{2 a(t)}\right)^{\prime}\right]=\frac{1}{2 a(t)} \text {. }
$$

This means that

$$
b(t)-2 a(t)-\frac{a^{\prime}(t)}{a(t)}=0 .
$$

Thus Eq. (50) gives

$$
z-\frac{1}{2 a(t)} x^{2}=c_{2} e^{-\int b(t) d t},
$$

where $c_{2}$ is a free parameter (constant of integration).

Now, substituting Eq. (53) into the second differential equation of system (49), we obtain

$$
\frac{d y}{d t}=r_{1}(t) x-y-\frac{1}{2 a(t)} x^{3}
$$

where $r_{1}(t)=r(t)-c_{2} e^{-\int b(t) d t}$.

Differentiating both sides of the first equation of system (49) with respect to $t$, taking in consideration Eq.(54) and in view of $y=x+\frac{1}{a(t)} \frac{d x}{d t}$, we obtain

$$
\frac{d^{2} x}{d t^{2}}+p(t) \frac{d x}{d t}+q(t) x=-\frac{1}{2} x^{3}
$$

where $p(t)=a(t)+1-\frac{a^{\prime}(t)}{a(t)}$ and $q(t)=a(t)\left(1-r_{1}(t)\right)$.

If we assume that

$$
a(t)+1-\frac{a^{\prime}(t)}{a(t)}=0
$$


and

$$
a(t)\left(1-r_{1}(t)\right)=c_{3},
$$

where $c_{3}$ is a constant.

Combining Eq. (52) with Eq. (56), we get

$$
b(t)=3 a(t)+1 \text {. }
$$

On the other hand, Eq. (56) read as

$$
a^{\prime}(t)=a(t)+a^{2}(t),
$$

which is a Bernoulli's equation and its solution reads as

$$
a(t)=\frac{c_{4} e^{t}}{1-c_{4} e^{t}} .
$$

Then Eq. (55) becomes

$$
\frac{d^{2} x}{d t^{2}}+c_{3} x+\frac{1}{2} x^{3}=0 .
$$

Multiplying both sides of Eq. (61) by $\frac{d x}{d t}$ and after some simple algebra we get

$$
\frac{d}{d t}\left[\left(\frac{d x}{d t}\right)^{2}+c_{3} x^{2}+\frac{x^{4}}{4}\right]=0 .
$$

Thus

$$
\left(\frac{d x}{d t}\right)^{2}+c_{3} x^{2}+\frac{x^{4}}{4}=c_{5}
$$

which can be solved for $\frac{d x}{d t}$, we obtain the separable differential equation

$$
\frac{d x}{d t}=\sqrt{c_{5}-c_{3} x^{2}-\frac{x^{4}}{4}}
$$

thus,

$$
\int \frac{d x}{\sqrt{4 c_{5}-4 c_{3} x^{2}-x^{4}}}=\frac{t}{2}+c_{6} .
$$

Therefore our result can be reformulated as

Lemma 2 If

$$
b(t)=3 a(t)+1
$$

and

$$
r(t)=c_{2} e^{-\int b(t) d t}+1-\frac{c_{3}}{a(t)}
$$

where

$$
a(t)=\frac{c_{4} e^{t}}{1-c_{4} e^{t}}
$$

Then the solution $(x(t), y(t), z(t))$ of system (49) is given as follows:

$x(t)$ can be found implicitly by Eq. (65), $z(t)$ can be obtained by Eq. (53) and $y(t)$ can be obtained from

$$
\frac{d y}{d t}=r(t) x-y-x z,
$$


that is

$$
y(t)=e^{-t} \int e^{t} \chi(t) d t+c_{1} e^{-t}, \chi(t)=r(t) x(t)-x(t) z(t) .
$$

\section{Discussion and Conclusion}

We have proposed a new method to refine the numerical methods to solve analytically a system of nonlinear coupled differential equations; i.e. the Lorenz equations by performing a special transformation and then attaining the Abel differential equations that can be solved analytically. The conditions of a total separation form an interesting field of investigation. The separation condition $a=2 b$ as it is stated in lemma (1) means that these solutions are not chaotic, since the condition of the existence of the chaotic solutions for the considered system is $a>b+1$. But the analytic form of the non chaotic solutions can be obtained by our proposed approach and the conditions of separation constitute an interesting subject of investigation. Indeed, the conditions of separation of Lorenz equations as they are stated in lemma (1) are $b=2 a$ and $r=1-\alpha / a$, which can be read in the case of the single mode laser equations

$$
\begin{aligned}
\frac{1}{T_{1}} & =\frac{2}{T_{c}} \\
w g T_{c} T_{2} & =\frac{13}{9}+\frac{2}{9}\left(\frac{T_{2}}{T_{c}}+\frac{T_{c}}{T_{2}}\right)
\end{aligned}
$$

The first equation means that for the probability difference decay time $T_{1}$ should be half of the cavity damping time $T_{c}$ that can be realized in the new technology construction of QED-cavity, while the coherence decay time $T_{2}$ is not conditioned. On the other hand, the second condition shows that the limitation on the coupling rate $g$ between the cavity field and the atom is far for the laser threshold, in which the gain rate equals the loss rate $w g T_{c} T_{2}=1$. However, in the process of dropping the problem from three variables to one variable we also reduce the possibility of encountering certain instabilities that one-dimensional equations cannot reveal. Furthermore, due to some inevitable losses in experiments, the initial conditions that can be practically realized offer an interesting argument in our separation approach. The present procedure shows that the system can be analytically solved for a class of initial conditions [25], which is given by Eq.(23) that can be read in terms of the parameters of the single-mode laser as

$$
-W(0)=\rho_{a a}(0)-\rho_{b b}(0)=1+\kappa \frac{T_{2}}{T_{c}} \frac{W_{s s}}{\Omega_{s s}} \Omega(0)
$$

where $\rho_{a a}, \rho_{b b}$ are the diagonal element of the density matrix that represent the probabilities in the ground and excited states. Finally, the separation approach of nonlinear coupled equations constitutes an important challenge in mathematics as well as in physics.

In general the investigation the conditions, which make the system separable or can be transformed to another system that can be solved analytically, is an interesting point of research. For these reasons, the Lorenz equations attract our intention to explore some 
analytic solution in order to avoid and reduce the numerical volume. We have presented a new parametric approach to solve this nonlinear coupled differential equations and an eventual extension for the case of variable coefficients. These results engender a series of questions of mathematical as well as physical consequence. Perfectly one would like to be capable to predict the behavior of paths for any set of initial conditions and parameter values. This is very much an interesting question in general. The Lorenz equations play an important task in the chaotic aspects, especially in laser physics and quantum optics, and have motivated the progress of techniques to explore more and more complicated and higher dimensional models.

\section{References}

[1] Uspenskii A V 1963 Radio Eng. Electron. Phys. (USSR) 81145.

[2] Buley E R and Cummings F W 1964 Phys. Rev. 134A 1454.

[3] Risken H and Nummedal K 1968 Phys. Lett. 26A 275.

[4] Meystre P and Sargent M III (eds) 1999 Elements of Quantum Optics, ${ }^{\text {rd }}$,(Springer-Verlag, Germany).

[5] Mandel L and Wolf E 1995 Optical Coherence and Quantum Optics, (Cambridge University Pres, USA).

[6] Ficek Z and Wahiddin M R 2004 Quantum Optics, Fundamentals and Applications, (Research Centre, International Islamic University Malaysia).

[7] Haken H 1985 Light, Vol.2, Laser Light Dynamics, (North-Holland Physics Publishing, Amesterdam).

[8] Haken H 1975 Phys. lett. 53 A 77.

[9] Lorenz E 1963 J. Atmospheric Sci. 20130.

[10] Mischaikow K and Mrozek M 1995 Bull. AMS 32136.

[11] Guckenheimer J and Holmes P 1983 Nonlinear Oscillations, Dynamical Systems, and Bifurcations of Vector Fields, 4th Edition, (Springer-Verlag NY).

[12] Sparrow C 1982 The Lorenz equations: bifurcations, chaos, and strange attractors, (SpringerVerlag, New York).

[13] Bougouffa S and Al-Awfi S 2008 Jour. Mod. Optics 55473

[14] Bougouffa S and Al-Awfi S 2007 Int. J. Theor. Phys. 46920

[15] Hopf E 1952 J. Rat. Mech. Anal , 1,87.

[16] Foias C 1973 Rend. Sem. Mat. Univ. Padova 499.

[17] Foias C, Jolly M S, kukavica I and Titi E S 2001 Discrete and Continuous Dynamical Systems 7 403.

[18] Yu P and Chen G 2004 International Journal of Bifrcation and Choas, 141683

[19] Szemplinska-Stupnicka W, Looss G and Moom F C 1988, Chaotic motions in nonlinear dynamics systems, (Springer Wien New York) .

[20] Bougouffa S 2008AIP Conf. Proc. 1048109

[21] Bougoffa L., Al-Awfi S and Bougouffa S 2007 Applied Mathematical Sciences 1, 2917

[22] Klische W and Weiss C O 1985 Phys. Rev. A 314049.

[23] Harrison R Al-Saidi I and Biswas D 1985 IEEE Journal of Quantum Electronic 211491.

[24] Polyanin A.D. and Zaitsev V F 2003 Handbook of exact solutions for ordinary differential equations (Boca Raton, FL: CRC Press)

[25] ShiJun L and PengFei W 2014 Sci. Chin. Phys., Mech. Astron. 57330 